\title{
Rickettsia felis
}

National Cancer Institute

\section{Source}

National Cancer Institute. Rickettsia felis. NCI Thesaurus. Code C86721.

A species of aerobic, Gram negative, rod and cocci shaped bacteria assigned to the phylum Proteobacteria. This species is motile, obligately intracellular and transmitted to humans by the bite of the cat flea, Ctenocephalides felis. R. felis is a pathogen that causes fever and may present other symptoms including headache, rash, vomiting, and stupor. 